Preprint typeset in JHEP style - HYPER VERSION

\title{
Remarks on self-interaction correction to black hole radiation
}

\author{
Qing-Quan Jiang ${ }^{a, b}$ and $\mathrm{Xu} \mathrm{Cai}^{b}$ \\ ${ }^{a}$ Institute of Theoretical Physics, China West Normal University, Nanchong, Sichuan \\ 637002, People's Republic of China \\ ${ }^{b}$ Institute of Particle Physics, Central China Normal University, Wuhan, Hubei 430079, \\ People's Republic of China \\ E-mail address: jiangqq@iopp.ccnu.edu.cn, xcai@mail.ccnu.edu.cn
}

\begin{abstract}
In the work [P. Kraus and F. Wilczek, Self-interaction correction to black hole radiation, Nucl. Phys. B433 (1995) 403], it has been pointed out that the selfgravitation interaction would modify the black hole radiation so that it is no longer thermal, where it is, however, corrected in an approximate way and therefore is not established its relationship with the underlying unitary theory in quantum theory. In this paper, we revisit the self-gravitation interaction to Hawking radiation of the general spherically symmetric black hole, and find that the precisely derived spectrum is not only deviated from the purely thermal spectrum, but most importantly, is related to the change of the BekensteinHawking entropy and consistent with an underlying unitary theory.
\end{abstract}

Keywords: Black holes, Modes of Quantum Gravity. 


\section{Contents}

1. Introduction

2. Eliminating the non-physical degrees of freedom for the particle-hole system 2

3. Self-interaction correction to Hawking radiation 7

4. Conclusion and Discussion 9

A. The ADM decomposition 9

B. The Schwarzschild black hole in the Painlevé coordinate 10

\section{Introduction}

In 1974, Hawking proved that black hole can radiate particles from its event horizon with a temperature proportional to its surface gravity, and the radiant spectrum is a purely thermal one [1], where the background geometry was approximately given by calculating the response of quantum fields to the collapse geometry. Now it is difficult to reconcile with the unitary evolution in quantum theory, which finally generates an information loss of black hole. In the classical theory, the loss of information was not a serious problem since the information could be thought of as preserved inside the black hole but just not very accessible. However, taking the quantum effect into consideration, the situation is changed. With the emission of thermal radiation, black holes could lose energy, shrink, and eventually evaporate away completely. Since the radiation with a precise thermal spectrum carries no information, the information carried by a physical system falling toward black hole singularity has no way to be recovered after a black hole has disappeared completely. This is the so-called "information loss paradox", which means that pure quantum states (the original matter that forms the black hole) can evolve into mixed states (the thermal spectrum at infinity)[2]. Such an evolution violates the fundamental principles of quantum theory, as these prescribe a unitary time evolution of basis states. To resolve the information loss paradox, one must go beyond the approximation of treating the background geometry as fixed, and treat it as a quantum variable and then quantize the gravitational system.

In 1995, Kraus and Wilczek found the black hole radiation is no longer thermal after considering its self-gravitation interaction [3]. Its idea is based on the Hamiltonian quantization, where the coupled particle-hole system is treated as the quantized system. 
After eliminating the non-physical degrees of freedom and quantizing the reduced system, the derived radiation deviates from the purely thermal spectrum. This elaborate work is invaluable due to the facts that: 1) it may provide a possible explanation to the problem of the information loss; 2) it can open a window to study the quantum gravity. Till now, the two open questions are still unsolved and present at the frontier of the modern theoretical physics. So it is necessary to find some new physics by solving the self-gravitational interaction on black hole radiation via the Hamiltonian quantization method.

In [3], it has been proved that the self-gravitational interaction would modify the black hole radiation, but the way in which it is corrected is not definite. In this paper, we revisit the self-gravitation interaction to Hawking radiation of the spherically symmetric black hole, and find that the precisely derived spectrum is not only deviated from the purely thermal spectrum, but most importantly, is related to the change of BekensteinHawking entropy and consistent with an underlying unitary theory. This result presents a definite way in which the black hole radiation is corrected as a result of its self-gravitational interaction, and moreover guarantees the unitary evolution in black hole quantum radiation.

The remainders of this paper are outlined as follows. In Sec.2, we first solve the constraint conditions to eliminate the non-physical degrees of freedom for the particle-hole system, and keep only those degrees of freedom which are most relevant to the problem of particle emission from the regions of low curvature. Sec.3 is to quantize the reduced particle-hole system, and present the self-interaction correction to black hole radiation. Sec. 1 ends up with some discussions and conclusions.

\section{Eliminating the non-physical degrees of freedom for the particle-hole system}

The four dimensional metric in the ADM(Arnowitt-Deser-Misner)(namely, (3+1) decomposition) form can be written as [5, 6]

$$
\begin{aligned}
d s^{2} & =-\left(\mathcal{N}^{2}\left(t, x^{i}\right)-\mathcal{N}_{i}\left(t, x^{i}\right) \mathcal{N}^{i}\left(t, x^{i}\right)\right) d t^{2} \\
& +2 \mathcal{N}_{i}\left(t, x^{i}\right) d x^{i} d t+h_{i j}\left(t, x^{i}\right) d x^{i} d x^{j}
\end{aligned}
$$

where $\mathcal{N} \equiv\left(-g^{00}\right)^{-1 / 2}$ is formally called as the time shift, $\mathcal{N}_{i} \equiv g_{0 i}$ as the space shift, and $\mathcal{N}^{i}=h^{i j} \mathcal{N}_{j}$. This decomposition for the gravitational field can be found in Appendix A. Now the new expression of the action for the shell-hole system in the ADM form can be written as

$$
\begin{aligned}
S & =\int d^{4} x \sqrt{h} \mathcal{N}\left(\mathcal{R}^{(3)}+\mathcal{K}_{i j} \mathcal{K}^{i j}-\mathcal{K}^{2}\right) \\
& -m \int d t \sqrt{\hat{\mathcal{N}}^{2}-\hat{\mathcal{N}}_{i} \hat{\mathcal{N}}^{i}-2 \hat{\mathcal{N}}_{i} \dot{\hat{x}}^{i}-\hat{h}_{i j} \dot{\hat{x}}^{i} \dot{\hat{x}}^{j}}+2 \int \sqrt{h} \mathcal{K} d^{3} x
\end{aligned}
$$

where $\mathcal{K}_{i j}=\frac{1}{2 \mathcal{N}}\left[\dot{h}_{i j}-\left(\mathcal{N}_{i \mid j}+\mathcal{N}_{j \mid i}\right)\right]$, in which $i \mid j$ is the covariant derivative on the metric $h_{i j}$, and $\mathcal{K}=h^{i j} \mathcal{K}_{i j}$. In addition, $\mathcal{R}^{(3)}$ denotes the scalar curvature of the 3 -dimensional spacial metric $h_{i j} . m$ is the rest mass of the shell, and the carets instruct one to evaluate 
physical quantities at the shell (as an example, $\hat{\mathcal{N}}=\mathcal{N}\left(\hat{t}, \hat{x}^{i}\right)$ ). Obviously, the independent variables for the action in the ADM form appear as $h_{i j}, \mathcal{N}$ and $\mathcal{N}_{i}$. Since Hamiltonian method is more flexible than Lagrangian method in eliminating constraints, in this paper we resort to Hamiltonian method to eliminate the non-physical degrees of freedom for the particle-hole system. To do so, we must first write the action (2.2) in the canonical form. In our case, we consider the the emitted particles coupled to the spherically symmetric gravitational fields, and its reduced 3-dimensional spacial line element is taken the form as

$$
d s_{3}^{2}=e^{2 \mu} d r^{2}+e^{2 \lambda}\left(d \theta^{2}+\sin \theta^{2} d \phi^{2}\right) .
$$

Assuming $\pi^{i j}$ is the canonical momentum with respect to the 3 -dimensional spacial metric $h_{i j}$, we have

$$
\pi^{r r}=\frac{1}{2} \pi_{\mu} e^{-2 \mu}, \quad \pi^{\theta \theta}=\frac{1}{4} \pi_{\lambda} e^{-2 \lambda}, \quad \pi^{\phi \phi}=\frac{1}{4} \pi_{\lambda} e^{-2 \lambda},
$$

where $\pi_{\mu}$ and $\pi_{\lambda}$ are the momentum conjugate to $\mu$ and $\lambda$. Now the action of the gravityshell system in the canonical form can be written as

$$
\begin{aligned}
S & =\int d t d r\left[\pi_{\mu} \dot{\mu}+\pi_{\lambda} \dot{\lambda}-\mathcal{N}\left(\mathcal{H}_{g}^{0}+\mathcal{H}_{s}^{0}\right)-\mathcal{N}_{r}\left(\mathcal{H}_{g}^{r}+\mathcal{H}_{s}^{r}\right)\right] \\
& +\int d t p_{r} \dot{\hat{r}}-\int d t M_{A D M},
\end{aligned}
$$

where

$$
\begin{aligned}
\mathcal{H}_{s}^{0} & =\sqrt{p_{r}^{2} e^{-2 \mu}+m^{2}} \delta(r-\hat{r}), \quad \mathcal{H}_{s}^{r}=-p_{r} e^{-2 \mu} \delta(r-\hat{r}), \\
\mathcal{H}_{g}^{0} & =e^{-\mu-2 \lambda}\left[\frac{1}{2} \pi_{\mu}^{2}-\pi_{\mu} \pi_{\lambda}+\frac{1}{2} e^{4 \lambda}\left(2 \lambda^{\prime \prime}-2 \lambda^{\prime} \mu^{\prime}+3 \lambda^{\prime}-e^{2(\mu-\lambda)}\right)\right], \\
\mathcal{H}_{g}^{r} & =-e^{-2 \mu}\left(\pi_{\mu}^{\prime}-\mu^{\prime} \pi_{\mu}-\lambda^{\prime} \pi_{\lambda}\right) .
\end{aligned}
$$

Here the last term in Eq.(2.5) is present to cancel the unwanted terms when carrying on the variation of the action [3]. The general relativity is a theory with constraints, so we wish to eliminate the gravitational degrees of freedom to obtain the effective action only depending on the shell variables by solving the constraints. Since the action (2.5) is not the functional of the parameters $\dot{\mathcal{N}}$ and $\dot{\mathcal{N}}_{r}$, the constraints are obtained by varying with respect to $\mathcal{N}$ and $\mathcal{N}_{r}$ as $\mathcal{H}^{0}=\mathcal{H}_{g}^{0}+\mathcal{H}_{s}^{0}=0$ (the energy constraint) and $\mathcal{H}^{r}=\mathcal{H}_{g}^{r}+\mathcal{H}_{s}^{r}=0$ (the momentum constraint). Obviously, the momentum $\pi_{\mu}$ and $\pi_{\lambda}$ can be obtained from the constraints. For simplicity, we, here, consider the linear combination of constraints as

$$
\begin{aligned}
0 & =e^{\lambda-\mu} \lambda^{\prime} \mathcal{H}^{0}+e^{-\lambda} \pi_{\mu} \mathcal{H}^{r} \\
& =-\frac{1}{2} \partial_{r}\left(e^{-2 \mu-\lambda} \pi_{\mu}^{2}+e^{\lambda}-e^{\lambda-2 \mu}\left(e^{\lambda} \lambda^{\prime}\right)^{2}\right) \\
& +\hat{e}^{\lambda-\mu} \hat{\lambda}^{\prime} \mathcal{H}_{s}^{0}+\hat{e}^{-\lambda} \hat{\pi}_{\mu} \mathcal{H}_{s}^{r} .
\end{aligned}
$$

We can obtain from the constraint (2.7) that at the shell $\hat{\pi}_{\mu}=0$ and $\hat{\lambda}^{\prime}=0$, which implies $\lambda^{\prime}$ is discontinuous here. Substituting the physical quantities at the shell into the energy constraint equation and integrating them, we have $\lambda^{\prime}(\hat{r}+\epsilon)-\lambda^{\prime}(\hat{r}-\epsilon)=\hat{e}^{-2 \lambda} \sqrt{p_{r}^{2}+m^{2} \hat{e}^{-2 \mu}}$. 
If we define $\mathcal{M} \equiv \frac{1}{2}\left(e^{-2 \mu-\lambda} \pi_{\mu}^{2}+e^{\lambda}-e^{\lambda-2 \mu}\left(e^{\lambda} \lambda^{\prime}\right)^{2}\right)$, it can be treated as the mass parameter when considering a static slice $\left(\pi_{\mu}=\pi_{\lambda}=0\right)$. Away from the shell, the constraints tell us $\mathcal{M}$ is a constant. At the shell, its presence causes $\mathcal{M}$ to be discontinuous. Here we take the forms of the discontinuous mass parameter in different regions as $\mathcal{M}=M$ at $(r<\hat{r})$, and $M=M_{+}$at $(r>\hat{r})$. As there is no matter outside the shell, we have $M_{A D M}=M_{+}$. Now the momentum $\pi_{\mu}$ can be written as

$$
\begin{array}{ll}
\pi_{\mu}=e^{\mu+\lambda} \sqrt{\left(e^{\lambda} \lambda^{\prime}\right)^{2} e^{-2 \mu}-1+2 M e^{-\lambda}}, & r<\hat{r}, \\
\pi_{\mu}=e^{\mu+\lambda} \sqrt{\left(e^{\lambda} \lambda^{\prime}\right)^{2} e^{-2 \mu}-1+2 M_{+} e^{-\lambda}}, & r>\hat{r} .
\end{array}
$$

When the constraints are satisfied, considering a variation of the action yields $d S=p_{r} d \hat{r}+$ $\int d r\left(\pi_{\mu} \delta \mu+\pi_{\lambda} \delta \lambda\right)-M_{+} d t$. To obtain the effective action, we must integrate the expression for an arbitrary shell trajectory. In our case, when holding $\hat{r}, p_{r}$ and $\lambda$ fixed, varying $\mu$ until $\pi_{\mu}=\pi_{\lambda}=0$ for an arbitrary trajectory yields

$$
\begin{aligned}
S & =\int_{r_{\text {min }}}^{\infty} d r \int_{0}^{\mu} \pi_{\mu} \delta \mu \\
& =\int_{r_{\min }}^{\hat{r}-\epsilon} d r\left[e^{\mu+\lambda} \sqrt{\left(e^{\lambda} \lambda^{\prime}\right)^{2} e^{-2 \mu}-1+2 M e^{-\lambda}}\right. \\
& \left.+e^{\lambda}\left(e^{\lambda} \lambda^{\prime}\right) \log \left|\frac{\left(e^{\lambda} \lambda^{\prime}\right) e^{-\mu}-\sqrt{\left(e^{\lambda} \lambda^{\prime}\right)^{2} e^{-2 \mu}-1+2 M e^{-\lambda}}}{\sqrt{\left|1-2 M e^{-\lambda}\right|}}\right|\right] \\
& +\int_{\hat{r}+\epsilon}^{\infty} d r\left[e^{\mu+\lambda} \sqrt{\left(e^{\lambda} \lambda^{\prime}\right)^{2} e^{-2 \mu}-1+2 M_{+} e^{-\lambda}}\right. \\
& \left.+e^{\lambda}\left(e^{\lambda} \lambda^{\prime}\right) \log \left|\frac{\left(e^{\lambda} \lambda^{\prime}\right) e^{-\mu}-\sqrt{\left(e^{\lambda} \lambda^{\prime}\right)^{2} e^{-2 \mu}-1+2 M_{+} e^{-\lambda}}}{\sqrt{\left|1-2 M_{+} e^{-\lambda}\right|}}\right|\right] .
\end{aligned}
$$

From Eq.(2.9), we can easily learn the variables are now turn to be $\mu, \lambda, \lambda^{\prime}$ and $M_{+}$. In the next stage, if an arbitrary variation of $\mu$ and $\lambda$ is carried on the effective action (2.9), it changes as

$$
\begin{aligned}
d S & =\int_{r_{\min }}^{\infty} d r\left(\pi_{\lambda} \delta \lambda+\pi_{\mu} \delta \mu\right) \\
& -\left[\frac{\partial S}{\partial \hat{\lambda}^{\prime}}(\hat{r}+\epsilon)-\frac{\partial S}{\partial \hat{\lambda}^{\prime}}(\hat{r}-\epsilon)\right] d \hat{\lambda}^{\prime}-\frac{\partial S}{\partial M_{+}} d M_{+}
\end{aligned}
$$

where the last two terms are present to keep the relations $\frac{\delta S}{\delta \lambda}=\pi_{\lambda}$ and $\frac{\delta S}{\delta \mu}=\pi_{\mu}$ unchanged when carrying on the arbitrary variations of $\lambda$ and $\mu$. Now we can additionally consider variations in the variables $p_{r}, \hat{r}$ and $t$ to obtain the final effective action, however it is no need to separately consider variations of $p_{r}$ and $\hat{r}$ the constraint equations contain their variations, since its contributions to the effective action has already been included in the constraints. As for the time variable $t$, its contribution to the action is $d S=-M_{+} d t$. 
Finally, the action can be written as

$$
\begin{aligned}
S & =\int_{r_{\text {min }}}^{\hat{r}-\epsilon} d r\left[e^{\mu+\lambda} \sqrt{\left(e^{\lambda} \lambda^{\prime}\right)^{2} e^{-2 \mu}-1+2 M e^{-\lambda}}\right. \\
& \left.+e^{\lambda}\left(e^{\lambda} \lambda^{\prime}\right) \log \left|\frac{\left(e^{\lambda} \lambda^{\prime}\right) e^{-\mu}-\sqrt{\left(e^{\lambda} \lambda^{\prime}\right)^{2} e^{-2 \mu}-1+2 M e^{-\lambda}}}{\sqrt{\left|1-2 M e^{-\lambda}\right|}}\right|\right] \\
& +\int_{\hat{r}+\epsilon}^{\infty} d r\left[e^{\mu+\lambda} \sqrt{\left(e^{\lambda} \lambda^{\prime}\right)^{2} e^{-2 \mu}-1+2 M_{+} e^{-\lambda}}\right. \\
& \left.+e^{\lambda}\left(e^{\lambda} \lambda^{\prime}\right) \log \mid \frac{\left.\left(e^{\lambda} \lambda^{\prime}\right) e^{-\mu}-\sqrt{\left(e^{\lambda} \lambda^{\prime}\right)^{2} e^{-2 \mu}-1+2 M_{+} e^{-\lambda}} \mid\right]}{\sqrt{\mid 1-2 M_{+} e^{-\lambda \mid}}}\right] \\
& -\int d t \dot{\hat{\lambda}} \hat{e}^{2 \lambda}\left[\log \left|\frac{\left(\hat{e}^{\lambda} \lambda^{\prime}(\hat{r}-\epsilon)\right) \hat{e}^{-\mu}-\sqrt{\left(\hat{e}^{\lambda} \lambda^{\prime}(\hat{r}-\epsilon)\right)^{2} \hat{e}^{-2 \mu}-1+2 M \hat{e}^{-\lambda}}}{\sqrt{\left|1-2 M \hat{e}^{-\lambda}\right|}}\right|\right. \\
& \left.-\log \left|\frac{\left(\hat{e}^{\lambda} \lambda^{\prime}(\hat{r}+\epsilon)\right) \hat{e}^{-\mu}-\sqrt{\left(\hat{e}^{\lambda} \lambda^{\prime}(\hat{r}+\epsilon)\right)^{2} \hat{e}^{-2 \mu}-1+2 M_{+} \hat{e}^{-\lambda}}}{\sqrt{\mid 1-2 M_{+} \hat{e}^{-\lambda \mid}}}\right|\right] \\
& \left.-\int d t \int_{\hat{r}+\epsilon}^{\infty} d r \frac{e^{\mu}}{\sqrt{\left(e^{\lambda} \lambda^{\prime}\right)^{2} e^{-2 \mu}-1+2 M_{+} e^{-\lambda}}} \mid\right] d t M_{+} \cdot
\end{aligned}
$$

Differentiating (2.11), we can successfully recover the variation of the action where the constraints are satisfied. This shows (2.11) is the correct expression of the action. At the shell, $\lambda^{\prime}$ is discontinuous, and its form arbitrarily near the shell is constrained by $\lambda^{\prime}(\hat{r}+\epsilon)-\lambda^{\prime}(\hat{r}-\epsilon)=\hat{e}^{-2 \lambda} \sqrt{p_{r}^{2}+m^{2} \hat{e}^{-2 \mu}}$. Near the shell but far enough away so that $\lambda^{\prime}$ can be well specifiable, we can define $\lambda_{f}^{\prime}=\lambda^{\prime}$. Arbitrarily near the horizon, we can well define $\lambda_{f}^{\prime}=\lambda^{\prime}(\hat{r}+\epsilon)$, and $\lambda^{\prime}(\hat{r}-\epsilon)$ can then be determined by the discontinuous constraint condition. The Lagrangian function is defined by the derivative of the action $S$ on the time $t$, which yields

$$
\begin{aligned}
\mathcal{L} & =\frac{d S}{d t}=\dot{\hat{r}} \hat{e}^{\mu+\lambda}\left[\sqrt{\left(\hat{e}^{\lambda} \lambda_{f}^{\prime}\right)^{2} \hat{e}^{-2 \mu}-1+2 M \hat{e}^{-\lambda}}\right. \\
& \left.-\sqrt{\left(\hat{e}^{\lambda} \lambda_{f}^{\prime}\right)^{2} \hat{e}^{-2 \mu}-1+2 M_{+} \hat{e}^{-\lambda}}\right] \\
& -\dot{\hat{\lambda}} \hat{e}^{2 \lambda} \log \left|\frac{\left(\hat{e}^{\lambda} \lambda^{\prime}(\hat{r}-\epsilon)\right) \hat{e}^{-\mu}-\sqrt{\left(\hat{e}^{\lambda} \lambda^{\prime}(\hat{r}-\epsilon)\right)^{2} \hat{e}^{-2 \mu}-1+2 M \hat{e}^{-\lambda}}}{\left(\hat{e}^{\lambda} \lambda_{f}^{\prime}\right) \hat{e}^{-\mu}-\sqrt{\left(\hat{e}^{\lambda} \lambda_{f}^{\prime}\right)^{2} \hat{e}^{-2 \mu}-1+2 M_{+} \hat{e}^{-\lambda}}}\right| \\
& +\int_{\min }^{\hat{r}-\epsilon} d r\left(\pi_{\lambda} \dot{\lambda}+\pi_{\mu} \dot{\mu}\right)+\int_{\hat{r}+\epsilon}^{\infty} d r\left(\pi_{\lambda} \dot{\lambda}+\pi_{\mu} \dot{\mu}\right)-M_{+} \cdot
\end{aligned}
$$

Considering the discontinuous constraint condition of $\lambda^{\prime}$ arbitrarily near the shell, and the 
shell mass approaching to infinite small, the Lagrangian function (2.12) becomes

$$
\begin{aligned}
\mathcal{L} & =\frac{d S}{d t}=\dot{\hat{r}} \hat{e}^{\mu+\lambda}\left[\sqrt{\left(\hat{e}^{\lambda} \lambda_{f}^{\prime}\right)^{2} \hat{e}^{-2 \mu}-1+2 M \hat{e}^{-\lambda}}\right. \\
& \left.-\sqrt{\left(\hat{e}^{\lambda} \lambda_{f}^{\prime}\right)^{2} \hat{e}^{-2 \mu}-1+2 M_{+} \hat{e}^{-\lambda}}\right] \\
& -\eta \dot{\hat{\lambda}} \hat{e}^{2 \lambda} \log \left|\frac{\left(\hat{e}^{\lambda} \lambda_{f}^{\prime}\right) \hat{e}^{-\mu}-\eta \sqrt{\left(\hat{e}^{\lambda} \lambda_{f}^{\prime}\right)^{2} \hat{e}^{-2 \mu}-1+2 M_{+} \hat{e}^{-\lambda}}}{\left(\hat{e}^{\lambda} \lambda_{f}^{\prime}\right) \hat{e}^{-\mu}-\eta \sqrt{\left(\hat{e}^{\lambda} \lambda_{f}^{\prime}\right)^{2} \hat{e}^{-2 \mu}-1+2 M \hat{e}^{-\lambda}}}\right| \\
& +\int_{\min }^{\hat{r}-\epsilon} d r\left(\pi_{\lambda} \dot{\lambda}+\pi_{\mu} \dot{\mu}\right)+\int_{\hat{r}+\epsilon}^{\infty} d r\left(\pi_{\lambda} \dot{\lambda}+\pi_{\mu} \dot{\mu}\right)-M_{+},
\end{aligned}
$$

where $\eta \equiv \pm$ corresponds to the momentum of the outgoing (ingoing) particle. Since at the black hole horizon, the particle radiates out, we should choose the + sign in the following discussion. Now the Lagrangian function has already been decided. To quantize the reduced particle-hole system we must first find the canonical coordinate (namely the physical degrees of freedom) and its corresponding canonical momentum. So we here must choose a gauge to specifically determine the form of the Lagrangian function (2.13). In this paper, we take the Schwarzschild black hole as an example to show in what definite way Hawking radiation is corrected due to self-gravitation interaction. The metric for a general spherically symmetric system in ADM form has been shown in Eqs.(2.1) and (2.3). For a four-dimensional spherically Schwarzschild solution $\mathcal{N}=1, \mathcal{N}_{i}=\sqrt{\frac{2 M}{r}}, e^{\mu}=1$ and $e^{\lambda}=r$. The Schwarzschild black hole in ADM form has been present in Appendix B. At the shell, $\hat{e}^{\lambda} \lambda^{\prime}$ is discontinuous, but in Eq. (2.13), $\hat{e}^{\lambda} \lambda_{f}^{\prime}$ is still freely specifiable. For the Schwarzschild black hole, the spacetime is static (namely, time independent), so the Lagrangian can be written as

$$
\mathcal{L}=\dot{\hat{r}}\left(\sqrt{2 M \hat{r}}-\sqrt{2 M_{+} \hat{r}}\right)-\dot{\hat{r}} \hat{r} \log \frac{\sqrt{\hat{r}}-\sqrt{2 M_{+}}}{\sqrt{\hat{r}}-\sqrt{2 M}}-M_{+}
$$

From Eq.(2.14), the canonical momentum conjugate to $\hat{r}$ is given by

$$
p_{\hat{r}}=\frac{\partial \mathcal{L}}{\partial \dot{\hat{r}}}=\sqrt{2 M \hat{r}}-\sqrt{2 M_{+} \hat{r}}-\hat{r} \log \frac{\sqrt{\hat{r}}-\sqrt{2 M_{+}}}{\sqrt{\hat{r}}-\sqrt{2 M}}
$$

which means the action for the particle-hole system in the canonical form can be written as

$$
S=\int d t \mathcal{L}=\int d t\left(p_{\hat{r}} \dot{\hat{r}}+p_{t}\right)
$$

where $p_{t}=M-M_{+}$comes from the contribution of the shell, and denotes the canonical momentum conjugate to the time $t$. Here we have omitted the term $\int d t M$, Since as a constant its contribution to the variation of the effective action can not change the physical result we want. Till now, we have eliminated the non-physical degrees of freedom for the particle-hole system, and obtained the effective action which only depended on the shell variables. In the next section, we focus on the quantization of the effective action and the self-interaction correction to Hawking radiation. 


\section{Self-interaction correction to Hawking radiation}

To pass to the quantum theory we should make the substitutions $p \rightarrow-i \frac{\partial}{\partial r}$ and $p_{t} \rightarrow-i \frac{\partial}{\partial t}$. But when returning to our case, how to implement this substitutions mets with factor ordering ambiguities. Fortunately, the mode solutions $\nu_{k}(t, r)$ to the field equations which are accurately described by the WKB approximation, are insensitive to these difficulties. Near the horizon, the infinite redshift results in a very short wavelength for the modes involved, so the WKB approximation is valid here. Therefore we can write these solutions as $\nu_{k}(t, r)=e^{i S(t, r)}$. Introducing the replacements $p_{\hat{r}} \rightarrow \frac{\partial S}{\partial \hat{r}}$ and $p_{t} \rightarrow \frac{\partial S}{\partial t}$, we can obtain the Hamilton-Jacobi equation for $S$. The solution of the the Hamilton-Jacobi equation is just the classical action. So, if $\hat{r}(t)$ is a classical trajectory derived by extremizing (2.16), we have

$$
S(t, \hat{r}(t))=S(0, \hat{r}(0))+\int_{0}^{t} d t\left(p_{\hat{r}}(\hat{r}(t)) \dot{\hat{r}}(t)+p_{t}\right) .
$$

In Eq. 3.1), as the initial condition $S(0, \hat{r}(0)) \equiv k \hat{r}(0)$, where $k$ must be arbitrarily large due to the ever increasing redshift experienced by the emitted quanta as they escape to infinity. As an arbitrary redshift existing at the horizon, when substituting (2.15) into (3.1) we can only keep the terms which is singular at the horizon. Thus we have

$$
S(t, r)=k \hat{r}(0)-\int_{\hat{r}(0)}^{r} d \hat{r} \hat{r} \log \frac{\sqrt{\hat{r}}-\sqrt{2 M_{+}}}{\sqrt{\hat{r}}-\sqrt{2 M}}+\left(M-M_{+}\right) t
$$

where

$$
k=\frac{\partial S}{\partial r}(0, \hat{r}(0))=-\hat{r}(0) \log \frac{\sqrt{\hat{r}(0)}-\sqrt{2 M_{+}}}{\sqrt{\hat{r}(0)}-\sqrt{2 M}} .
$$

For the Schwarzschild black hole, the black hole horizon is coincident with the infinite redshift surface. So at the horizon, $k \rightarrow \infty$, which yields from Eq.(3.3)

$$
\hat{r}(0)=2 M_{+} \pm \epsilon,
$$

where $\epsilon$ is a small constant, "+" corresponds to $M_{+}=M+\omega_{k}$, and "-" to $M_{+}=M-\omega_{k}$. Next, we focus on studying the emission rate of the black hole, including effects due to self-interaction. Black hole radiance originates from the mismatch between the two natural vacuum states which arise in the quantization of a field propagating on a black hole spacetime. The one is the vacuum state which is natural from the standpoint of an observer making measurements at the infinity, and the other vacuum state to consider is the one naturally employed by an observer freely falling through the horizon. As for the first vacuum state, we consider the modes which are positive frequency with respect to the Killing vector. We write these modes as $\mu_{k}(r) e^{-i \omega_{k} t}$. Thus the field operator can be expanded in these modes as

$$
\phi=\int d k\left(a_{k} \mu_{k}(r) e^{-i \omega_{k} t}+a_{k}^{\dagger} \mu_{k}^{*}(r) e^{i \omega_{k} t}\right) .
$$

Here the freely falling observer would find an infinite energy momentum density, since the modes $\mu_{k}(r)$ are singular at the horizon. In this case, the vacuum state is defined by 
$a_{k}\left|0_{\mu}\right\rangle=0$. To well describe the physical quantities at the horizon, this vacuum state is not expected, and the other vacuum state which is well behaved at the horizon is required. Writing the complete set of such modes by $\nu_{k}(t, r)$, the field operator can also be expanded as

$$
\phi=\int d k\left(b_{k} \nu_{k}(t, r)+b_{k}^{\dagger} \nu_{k}^{*}(t, r)\right),
$$

where the vacuum state is determined by $b_{k}\left|0_{\nu}\right\rangle=0$. This vacuum state $\left|0_{\nu}\right\rangle$ is well behaved at the horizon, so it can result in a finite energy-momentum density here. The operators in Eqs.(3.5) and (3.6) are related by the Bogoliubov coefficients as $a_{k}=\int d k^{\prime}\left(\alpha_{k k^{\prime}} b_{k}+\beta_{k k^{\prime}} b_{k^{\prime}}^{*}\right)$, where

$$
\begin{aligned}
\alpha_{k k^{\prime}} & =\frac{1}{2 \pi \mu_{k}(r)} \int_{-\infty}^{\infty} d t e^{i \omega_{k} t} \nu_{k^{\prime}}(t, r), \\
\beta_{k k^{\prime}} & =\frac{1}{2 \pi \mu_{k}(r)} \int_{-\infty}^{\infty} d t e^{i \omega_{k} t} \nu_{k^{\prime}}^{*}(t, r) .
\end{aligned}
$$

These coefficients in (3.7) can be obtained in the saddle point approximation. As $\nu_{k}=$ $e^{i S(t, r)}$, the saddle point for $\alpha_{k k^{\prime}}$ is given by $\omega_{k}=-\partial_{t} S(t, r)=M_{+}-M$. From Eq.(3.4), this condition produces the integration region of the effective action $S$ to lie in between $\hat{r}(0)=2\left(M+\omega_{k}\right)+\epsilon$ and outside the place $\hat{r}(0)$. After integrating Eq. (3.2) in this region, we will find the action appears purely real, which yields $\left|\alpha_{k k^{\prime}}\right|^{2}=\frac{1}{\left|2 \pi \mu_{k}(r)\right|^{2}}$ in the saddle approximation. For $\beta_{k k^{\prime}}$, the saddle point is determined by $\omega_{k}=\partial_{t} S(t, r)=M-M_{+}$, which present an imaginary part for the action. In fact, if we assume that the mass of the Schwarzschild black hole is held fixed whereas the total ADM mass of the hole-particle system are allowed to fluctuate, when radiating a particle with the energy " $-\omega_{k}$ " from the black hole horizon, we also obtain the ADM energy $M_{+}=M-\omega_{k}$. At this point, we have

$$
\left|\beta_{k k^{\prime}}\right|^{2}=\frac{1}{\left|2 \pi \mu_{k}(r)\right|^{2}} \exp \left(2 \omega_{k} \operatorname{Im} t+2 \operatorname{Im}\left[S_{k}^{*}(t)\right]\right) .
$$

The emission rate is given by the effective Boltzmann factor as

$$
\begin{aligned}
\left|\frac{\beta_{k k^{\prime}}}{\alpha_{k k^{\prime}}}\right|^{2} & =e^{2 \operatorname{Im} \int_{\hat{r}(0)}^{r} d \hat{r} \hat{r} \log \frac{\sqrt{\hat{r}}-\sqrt{2\left(M-\omega_{k}\right)}}{\sqrt{\hat{r}}-\sqrt{2 M}}} \\
& =e^{4 \pi\left[\left(M-\omega_{k}\right)^{2}-M^{2}\right]}=e^{\Delta S_{B H}},
\end{aligned}
$$

where $\Delta S_{B H}=S_{B H}\left(M-\omega_{k}\right)-S_{B H}(M)$ is the change of the Bekenstein-Hawking entropy. So the emission rate is related to the Bekenstein "CHawking entropy, and the true radiation spectrum of the black hole deviates from the strictly thermal one. When considering the emission particle having a small energy, we can also expand Eq.(3.9) in terms of the energy $\omega_{k}$, and reproduce the purely thermal spectrum.

Note that Eq.(3.9) agrees with the underlying unitary theory since the emission rate in quantum mechanics is expressible as $\Gamma(i \rightarrow f) \propto\left|M_{f i}\right|^{2}$ (phase space factor), where $\left|M_{f i}\right|^{2}$ is the square of the amplitude for the emission process, and the phase-space factor is given by summing over the final states and averaging over the initial states. Since the number of the initial/final states is the exponent of the initial/final entropy, the emission rate is 
then given by $\Gamma(i \rightarrow f) \propto \frac{e^{S_{\text {final }}}}{e^{S_{\text {initial }}}}=e^{\Delta S}$, which is consistent with our result (3.9). Hence, Eq.(3.9) satisfies the underlying unitary theory and provides a right correction to Hawking precisely thermal radiation spectrum.

\section{Conclusion and Discussion}

In this paper, we revisit the self-gravitation correction to black hole radiation, and find that the precisely derived spectrum is not only deviated from the purely thermal spectrum (already present in Ref.[2]), but most importantly, is related to the change of the Bekenstein-Hawking entropy and consistent with an underlying unitary theory. The results are useful, as a starting point, for the quantization of the gravity, and guarantees the unitary evolution in black hole quantum radiation. Supposing the emitted particle taking an small energy, we can also recover Hawking purely thermal spectrum by expanding the result (3.9) to the first order.

Here, we have assumed that the mass of black hole is held fixed whereas the total ADM mass of the hole-particle system are allowed to fluctuate. Instead, we also fix the total ADM mass and allow the black hole mass to fluctuate. In Ref.[7], Parikh and Wilczek adopted the latter assumption to develop this method, and implemented Hawking radiation as a tunnelling process (later on, many papers appear to discuss Hawking radiation via tunnelling from different black holes [8, 9, 10, 11, 12, 13, 14]). In the two cases, we can reproduce the same results, and provide a correction to black hole radiation due to self-gravitation interaction. On the other hand, the emission rate (3.9) is semiclassically derived by applying the WKB approximation and the saddle point approximation. Such an approximation can only be valid in the low energy regime. If we are to properly describe Hawking radiation, then a better understanding of quantum gravity is a necessary prerequisite.

\section{A. The ADM decomposition}

The Hamilton description for general relativity demands a $(3+1)$-dimensional decomposition for the 4 -dimensional space-time. When the arbitrary point $\left(t, x^{i}\right)$ on the 3 -dimensional space-like surface moves to the point $\left(t+d t, x^{i}\right)$ after the time past $(t+d t)$, the surface would de deformed. Now, we can define the deformation vector as $\mathcal{N}^{\mu} \equiv \frac{\partial X^{\mu}\left(t, x^{i}\right)}{\partial t}$, where $X^{\mu}\left(t, x^{i}\right)$ is the space-like hypersurface. In $\left(t, x^{i}\right)$, the tangential vector defined by $X_{i}^{\mu} \equiv \frac{\partial X^{\mu}}{\partial x^{i}}$ and the longitudinal vector $n^{\mu}$ should satisfy

$$
g_{\mu \nu} X_{i}^{\mu} n^{\nu}=0, \quad g_{\mu \nu} X_{i}^{\mu} X_{j}^{\nu}=h_{i j}, \quad g_{\mu \nu} n^{\mu} n^{\nu}=-1 .
$$

The first term denotes the tangential vector and the longitudinal vector are orthogonal each other. The induce metric $h_{i j}$ for the arbitrary point on the space-like hypersurface is determined by the second term. The third term implies the longitudinal vector $n^{\mu}$ is time-like. The tangential vector $X_{i}^{\mu}$ and the longitudinal vector $n^{\mu}$ constitutes the local tetrad on the space-like hypersurface. Now making a $(3+1)$-dimensional decomposition for 
the deformation vector on the local tetrad, we have

$$
\mathcal{N}^{\mu}=\mathcal{N} n^{\mu}+\mathcal{N}^{i} X_{i}^{\mu}
$$

where $\mathcal{N}$ is called as the time shift, $\mathcal{N}_{i}$ as the space shift. For the general metric taking the form as

$$
d s^{2}=g_{t t} d t^{2}+2 g_{i t} d x^{i} d t+g_{i j} d x^{i} d x^{j}
$$

Noted that the deformation vector defined above means $\delta X^{\mu}=\mathcal{N}^{\mu} d t$, which yields

$$
g_{\mu \nu} \delta X^{\mu} \delta X^{\nu}=\mathcal{N}^{\mu} \mathcal{N}_{\mu} d t^{2}=\left(\mathcal{N}^{i} \mathcal{N}_{i}-\mathcal{N}^{2}\right) d t^{2}=g_{t t} d t^{2}
$$

where $\mathcal{N}_{i}=h_{i j} \mathcal{N}^{j}$. Similarly, for the component $g_{i t}$, we have

$$
g_{i t}=g_{\mu \nu} X_{i}^{\mu} \mathcal{N}^{\nu}=h_{i j} \mathcal{N}^{j}=\mathcal{N}_{i}
$$

Now the action for the (3+1)-dimensional gravitational field can be found by Eq.(2.2).

\section{B. The Schwarzschild black hole in the Painlevé coordinate}

The Schwarzschild black hole in the Schwarzschild coordinate can be written as

$$
d s^{2}=-\Delta d t_{s}^{2}+\Delta^{-1} d r^{2}+r^{2}\left(d \theta^{2}+\sin \theta^{2} d \phi^{2}\right),
$$

where $\Delta=\left(1-\frac{2 M}{r}\right)$. Introducing the Painlevé coordinate transformation as

$$
d t_{s}=d t+\frac{\sqrt{1-\Delta}}{\Delta}
$$

we have

$$
d s^{2}=-\Delta d t^{2}+2 \sqrt{1-\Delta} d t d r+d r^{2}+r^{2}\left(d \theta^{2}+\sin \theta^{2} d \phi^{2}\right) .
$$

The new metric has many attractive features. (i) The metric is regular at the event horizon;

(ii) The time coordinate $t$ registers the local proper time for radially free-falling observers;

(iii) In addition, it satisfies Landau's condition of the coordinate clock synchronization. Integrating the Painlevé coordinate transformation (B.2) yields

$$
t_{s}=t-2 \sqrt{2 M r}-2 M \log \frac{\sqrt{r}-\sqrt{2 M}}{\sqrt{r}+\sqrt{2 M}} .
$$

\section{Acknowledgments}

This work is supported by National Natural Science Foundation of China with Grant Nos.10675051, 70571027, 10635020, a grant by the Ministry of Education of China under Grant No.306022, and a Graduate Innovation Foundation by Central China Normal University. 


\section{References}

[1] S. W. Hawking, Black hole explosions, Nature. 30 (1974) 248

[2] S.W. Hawking, Particle creation by black hole, Commun. Math. Phys. 43 (1975)199

[3] P. Kraus and F. Wilczek, Self-interaction correction to black hole radiation, Nucl. Phys. B433 (1995) 403; Effect of self-interaction on charged black hole radiance, Nucl. Phys. B437 (1995) 231

[4] T. Regge and C. Teitelboim, Role of Surface Integrals in the Hamiltonian Formu- lation of General Relativity, Ann. Phys. 88 (1974) 286

[5] R. Arnowitt, S. Deser and C. W. Misner, The Dynamics of General Relativity, arXiv: gr-qc/0405109

[6] L, Liu and Z, Zhao, General Relativity, 2nd edition (Higher Education Press, Beijing, (2004))

[7] M. K. Parikh and F. Wilczek, Hawking Radiation As Tunneling, Phys. Rev. Lett. 85 (2000) 5042

[8] M. K. Parikh, A secret tunnel through the horizon, Int. J. Mod. Phys. D13 (2004) 2351; A secret tunnel through the horizon, Gen. Relativ. Gravit. 36 (2004) 2419;

K. Srinivasan and T. Padmanabhan, Particle production and complex path analysis, Phys. Rev. D60 (1999) 024007;

S. Shankaranarayanan, K. Srinivasan, and T. Padmanabhan, Method of complex paths and general covariance of Hawking radiation, Mod. Phys. Lett. A16 (2001) 571;

S. Shankaranarayanan, T. Padmanabhan, and K. Srinivasan, Hawking radiation in different coordinate settings: Complex paths approach, Class. Quant. Grav. 19 (2002) 2671

[9] S. Hemming and E. Keski-Vakkuri, Hawking radiation from AdS black holes, Phys. Rev. D64 (2001) 044006;

A. J. M. Medved, Radiation via tunneling from a de Sitter cosmological horizon, Phys. Rev. D66 (2002) 124009; Radiation via tunneling in the charged BTZ black hole, Class. Quant. Grav. 19 (2002) 589;

E. C. Vagenas, Are extremal $2 D$ black holes really frozen?, Phys. Lett. B503 (2001) 399; Generalization of the KKW analysis for black hole radiation, Phys. Lett. B559 (2003) 65;

Two-dimensional dilatonic black holes and Hawking radiation, Mod. Phys. Lett. A17 (2002) 609.

A. J. M. Medved and E. C. Vagenas, On Hawking radiation as tunneling with logarithmic corrections, Mod. Phys. Lett. A20 (2005) 1723; On Hawking radiation as tunneling with back-reaction, Mod. Phys. Lett. A20 (2005) 2449;

M. Arzano, A. J. M. Medved and E. C. Vagenas, Hawking radiation as tunneling through the quantum horizon, JHEP. 0509 (2005) 037.

[10] J. Y. Zhang and Z. Zhao, New coordinates for Kerr-Newman black hole radiation, Phys. Lett. B618 (2005) 14; Hawking radiation of charged particles via tunneling from the Reissner-Nordstroem black hole, JHEP 0505 (2005) 055;

Y. P. Hu, J. J. Zhang and Z. Zhao, Massive particles-prime Hawking radiation via tunneling from the G.H. dilaton black hole, Mod. Phys. Lett. A21 (2006) 2143;

C. Z. Liu, J. Y. Zhang and Z. Zhao, Charged particle's tunneling from a dilaton black hole, Phys. Lett. B639 (2006) 670; 
W. B. Liu, New coordinates for BTZ black hole and Hawking radiation via tunnelling, Phys. Lett. B634 (2006) 541

[11] Q. Q. Jiang and S. Q. Wu, Hawking radiation of charged particles as tunneling from Reissner-Nordstrom-de Sitter black holes with a global monopole, Phys. Lett. B635 (2006) 151;

Q. Q. Jiang, S. Q. Wu and X. Cai, Hawking radiation as tunneling from the Kerr and Kerr-Newman black holes, Phys. Rev. D73 (2006) 064003;

S. Q. Wu and Q. Q. Jiang, Remarks on Hawking radiation as tunneling from the BTZ black holes, JHEP. 0603 (2006) 079; Hawking radiation of charged particles as tunneling from higher dimensional Reissner-Nordstrom-de Sitter black holes, hep-th/0603082;

L. Zhao, Tunnelling through black rings, arXiv: hep-th/0602065.

[12] S. P. Kim, Hawking Radiation as Quantum Tunneling in Rindler Coordinate, JHEP. 0711 (2007) 048;

S. Sarkar and D. Kothawala, Hawking radiation as tunneling for spherically symmetric black holes: A Generalized treatment, Phys. Lett. B659 (2008) 683;

T. Pilling, Tunneling derived from Black Hole Thermodynamics, Phys. Lett. B660 (2008) 402;

M. H. Ali, Hawking radiation via tunneling from hot NUT-Kerr-Newman-Kasuya spacetime, Class. Quant. Grav. 24 (2007) 5849;

P. Mitra, Hawking Temperature From Tunnelling Formalism, Phys. Lett. B648 (2007) 240;

Z. Z. Ma, Hawking Temperature of Kerr-Newman-AdS black hole from tunneling, Phys. Lett. B666 (2008) 376;

B. C. Zhang, Q. Y. Cai and M. S. Zhan, Hawking radiation as tunneling derived from Black Hole Thermodynamics through the quantum horizon, Phys. Lett. B665 (2008) 260;

C. K. Ding and J. L. Jing, What kinds of coordinate can keep the Hawking temperature invariant for the static spherically symmetric black hole?, Class. Quant. Grav. 25 (2008) 145015 ;

Y. Sekiwa, Decay of the cosmological constant by Hawking radiation as quantum tunneling, arXiv:0802.3266[hep-th];

R. Kerner and R. B. Mann, Tunnelling, Temperature and Taub-NUT Black Holes, Phys. Rev. D73 (2006) 104010; Tunnelling From Gödel Black Holes, Phys. Rev. D75 (2007) 084022

[13] R. Kerner and R. B. Mann, Fermions Tunnelling from Black Holes, Class. Quant. Grav. 25 (2008) 095014; Charged Fermions Tunnelling from Kerr-Newman Black Holes, Phys. Lett. B665 (2008) 277;

A. Alexandre and R. B. Mann, Gravitinos Tunneling from Black Holes, Phys. Lett. B673 (2009)168;

R. Li and J. R. Ren, Dirac particles tunneling from BTZ black hole, Phys. Lett. B661 (2008) 370 ;

R. Li, J. R. Ren and S. W. Wei, Hawking radiation of Dirac particles via tunneling from Kerr black hole, Class. Quant. Grav. 25 (2008) 125016;

R. Di. Criscienzo and L. Vanzo, Fermion Tunneling from Dynamical Horizons, Europhys. Lett. 82 (2008) 60001;

D. Y. Chen, Q. Q. Jiang and X. T. Zu, Hawking radiation of Dirac particles via tunnelling from rotating black holes in de Sitter spaces, Phys. Lett. B665 (2008) 106; Fermions tunnelling from the charged dilatonic black holes, Class. Quant. Grav. 25 (2008) 205022; 
Q. Q. Jiang, Dirac particles' tunnelling from black rings, Phys. Rev. D78 (2008) 044009; Fermions tunnelling from GHS and non-extremal D1-D5 black holes, Phys. Lett. B666 (2008) 517

[14] R. Banerjee and B. R. Majhi, Quantum Tunneling Beyond Semiclassical Approximation, JHEP. 0806 (2008) 095;

B. R. Majhi, Fermion Tunneling Beyond Semiclassical Approximation, Phys. Rev. D79 (2009) 044005 ;

J. Y. Zhang, Black hole quantum tunnelling and black hole entropy correction, Phys. Lett. B668 (2008) 353 